\title{
UN'AMBASCIATA VENETA A CLARENZA PRESSO CATERINA DI VALOIS NEL 1341, DA DOCUMENTI INEDITI
}

\begin{abstract}
ANGELIKI TZAVARA ${ }^{1}$
Riassunto: Nel 1341, un'ambasciata veneta giunse a Clarenza presso l'imperatrice latina titulare di Costantinopoli Caterina di Valois, la quale si trovava in Morea, assieme al figlio Roberto, per consolidare il proprio potere. Le cause dell' invio della missione diplomatica erano state le continue proteste del Veneziani per il clima d'intolleranza e per l'erosione degli antichi privilegi commerciali. Gli obiettivi dell'ambasciata erano sì chiedere gli indennizi, ma soprattutto riaffermare nei confronti dei nuovi signori del principatoi privilegi già goduti dai mercanti. Diplomazìa.

Parole chiavi: Catherine de Valois; Clarenza; Venezia; Morea/Peloponeso;
\end{abstract}

Abstract: In 1341, a venetian ambassade arrived at Clarenza in order to meet the only Empress in name latin, Catherine de Valois, who was with her son Robert in Morea, so as to consolidate her power. The reason of the diplomatic mission was the continuous protestations made by the Venetians against the intollerance and and the erosion of their ancient commercial privileges. The objectives of the ambassade were no only to demand a compensation but, above all, to ask for the reaffirmation of the merchants'privileges by the new lords of the Principate.

Keywords: Catherine of Valois; Clarenza; Venezia; Morea/Peloponeso; Diplomacy.

Clarenza era una delle città più importanti del principato di Acaia insieme a Patrasso e Andravida. Sembra che essa sia stata costruita nella seconda metà del XIII secolo. Era situata nelle terre che appartenevano al principe di Morea, e dotata di un comodo porto, era stata frequentata fin dal tempo della sua fondazione dagli attivissimi mercanti provenienti dai diversi stati della penisola italiana, come fiorentini, senesi, napoletani e altri, soprattutto veneziani ${ }^{2}$.

\footnotetext{
${ }^{1}$ Università di Paris-Sorbonne. 2003.

Data di recezione dell'articolo: luglio 2003. Data di accettazione e versione finale: agosto
}

${ }^{2}$ Su Clarentza vedi A. BoN, La Morée franque. Recherches historiques, topographiques et archéologiques sur la Principauté d'Achaie, 1205-1430, Paris 1969; E. SARANTI-MENDELOVICI, $H \mu \epsilon \sigma \alpha \imath \omega \nu \iota \kappa \eta \Gamma \lambda \alpha \rho \epsilon \nu \tau \zeta \alpha$, "Diptycha", 2 (1980), pp. 61-71 ; O. JENS SCHMITT, Zur Geschichte

«Anuario de Estudios Medievales», 33/2 (2003), pp.905-926 .- ISSN 0066-5061. 
Quest'ultimi, secondo quanto convenuto nel trattato di Sapienza, avevano diritto a commerciare nell'intero principato liberamente, con privilegi fiscali e con la sicurezza per le persone e le merci garantita dalle autorità. Inoltre, essi potevano avere in tutte le città una chiesa, una corte e un fondaco $^{3}$. Questi eccezionali vantaggi commerciali avevano molte volte suscitato l'invidia della popolazione locale, la quale con la complicità di alcune autorità del principato, cercarono di danneggiare il commercio veneziano durante tutto il XIV secolo.

I Veneziani intendevano approfittare dei loro privilegi sin dall' inizio. In una deliberazione del Maggior Consiglio datata 1297, vi è la prima testimonianza dell'invio di un console veneziano a Clarenza ${ }^{5}$. Circa 40 anni più tardi, cioè nel 1339, i mercanti veneti Marco Falier, Franceschino Loredan, Moretto Gradenigo e Nicoletto Nani con altri Veneziani che soggiornavano e facevano commercio a Clarenza, chiedevano al Senato il permesso di costruire una chiesa dedicata a San Marco e una loggia per il ristoro dei mercanti su un terreno concesso dai frati minori di Clarenza ${ }^{6}$.

Ritorniamo un po' indietro nel tempo, e precisamente nel 1332, anno in cui il principato d'Acaia cambiò signore. Il principe Giovanni d'Angiò di Gravina cedeva il possesso del principato alla moglie di suo fratello Filippo d'Angiò, Caterina, in cambio dei titoli di duca di Durazzo e re dell'Albania. Caterina di Valois, aveva il titolo di imperatrice latina di Costantinopoli, e all'epoca era vedova. Essa agiva in nome di suo figlio primogenito Roberto, ancora infante. Secondo l'accordo di scambio, Caterina doveva a Giovanni cinque mila once d'oro, somma che fu interamente pagata nel 1338. Solo in questo momento Caterina e suo figlio potevano entrare a pieno titolo nel

der Stadt Glarentza im 15. Jahrhundert, "Byzantion", LXV/1 (1995), pp. 98-135.

${ }^{3}$ Il trattato, detto di Sapienza dall'isola dove esso era stato firmato nel luglio del 1209. Il trattato regolava le relazioni di Venezia con il principato della Morea. G.L. Fr. TAFEL-G.M. THOMAS, Urkuden zur Älteren Handels und Staatsgeschichte der Republik Venedig mit Besonder Beziehung auf Byzanz und die Levante, vol. II, Vienna, 1856, ed. anast. Amsterdam, 1964, pp. 96$100 \mathrm{n}^{\circ}$ CCVII, («salvi et securi esse debent in personis et rebus sine aliqua datazione, ... debent habere ecclesiam, fondiculum et curiam»).

${ }^{4}$ Tutte queste informazioni si trovano nella mia tesi di dottorato col titolo: Clarentza: une ville de la Morée latine (seconde moitié du XIII siècle-1428) che sarà discussa prossimamente.

${ }^{5} \mathrm{R}$. CESSI (a cura di), Deliberazioni del Maggior Consiglio di Venezia, vol. 3, Bologna, 1931 , p. $431, \mathrm{n}^{\circ} 57$.

${ }^{6} \mathrm{ASV}$, Senato, Misti, reg. 18 , f. 60 r.

«Anuario de Estudios Medievales», 33/2 (2003), pp.905-926 .- ISSN 0066-5061. 
possesso del principato. Caterina amministrava indirettamente, tramite dei baili. Nel 1336, fu nominato bailo di Morea, Bertrando des Baux?

Questi sei anni di ambiguità furono segnati da molte proteste, disordini e altri episodi gravi. Uno di questi era il conflitto tra il bailo e l'arcivescovo di Patrasso Guglielmo Frangipani. Questi rifiutò di prestare omaggio ai nuovi signori del principato dichiarando la diocesi indipendente ${ }^{8}$. Dopo la sua morte, avvenuta tra il 18 dicembre 1336 e il 20 ottobre 1337, il bailo mise l'assedio davanti la città di Patrasso'. Quest'ultimo termine era la data in cui il papa Benedetto XII aveva confermato l'elezione del nuovo arcivescovo Ruggero. Qualche giorno prima, il 26 settembre 1337, il papa aveva scritto a Caterina sulla questione di Patrasso, affermando che la diocesi doveva dipendere direttamente dalla Santa Sede ${ }^{10}$.

In questo contesto, Caterina decise di recarsi di persona nel principato, accompagnata dal figlio Roberto e dal suo consigliere Nicolò Acciaiuoli, per consolidare la propria signoria. Nicolò Acciaiuoli aveva acquistato e ottenuto in dono dall'imperatrice e dalla compagnia Acciaiuoli, numerose terre in Morea ${ }^{11}$. Di conseguenza, egli era interessato al futuro dei suoi possedimenti e al potere di Caterina e di Roberto in Morea. L'imperatrice era partita per la Morea, sicuramente dopo il 24 luglio 1338, data nella quale suo cognato il re di Napoli Roberto, aveva ordinato la preparazione del viaggio ${ }^{12}$. Probabilmente, Caterina giunse a Clarenza prima della fine dell'estate.

\footnotetext{
${ }^{7}$ A. Bon, La Morée franque, op. cit., pp. 208-209.

${ }^{8}$ A. Bon, La Morée franque, op. cit., pp. 211-212. E' da ricordare che l'arcivescovo di Patrasso era barone e quindi feudatario del principe.

${ }^{9}$ E. GERLAND, Neue Quellen zur Geschichte des lateinischen Erzbistums, Leipzig 1903, pp.

${ }^{10} \mathrm{~A}$. BoN, La Morée franque, op. cit., p. 212; G. FEDALTo, La chiesa latina in Oriente, vol. 1 , Verona 1981, p. 358.

${ }^{11}$ Su Nicolò Acciaiuoli vedi L. TANFANI, Niccola Acciaiuoli: Studi storici, Firenze, 1863; A. Bon, La Morée franque, op. cit., pp. 209-211; Dizionario Biografico degli Italiani, vol. 1, pp. 87-90 (con bibliografia).

${ }^{12}$ A. Bon, La Morée franque, op. cit., p. 209, n. 2. La versione aragonese della Cronaca di Morea menziona il viaggio di Caterina, A. MOREL-FATIO, Libro de los fechos et conquistas de principato de la Morea, Ginevra, 1885, p. 148: «e despues poco tempo fue madama la emperadriç (fue) en la Morea e fue en el anyo de mil e CCC.XXX.VII e levó en su companya á su fío micer Loys, sus fiias madama Margarita madama Maria; e estuuo en la Morea tres anyos». 26-27. 
Qualche tempo più tardi, ma sicuramente dopo il 10 ottobre dello stesso anno, partì anche Nicolò per raggiungere l'imperatrice in Morea ${ }^{13}$.

Caterina dunque desiderava, o almeno così sembrerebbe, mettere ordine al principato e garantire il futuro del suo primogenito. Non ci sono tante testimonianze sul suo soggiorno nel principato, ma grazie ad alcuni documenti inediti dell'Archivio di Stato di Venezia è possibile ricostruire alcune fasi delle relazioni tra la repubblica di San Marco e l'imperatrice.

Verso la fine del 1340, arrivarono al Senato veneto notizie, secondo le quali il console di Clarenza e alcuni mercanti veneti avevano subito dei danni e dei maltrattamenti da parte di autorità, ufficiali e sudditi dell'imperatrice. Non sappiamo quando questi episodi si svolsero ma le proteste coincidono col soggiorno di Caterina in città. In questo caso, come si spiega la richiesta di costruzione di un fondaco e una chiesa fatta dai Veneziani di Clarenza, al Senato, nel 1339? Questa richiesta testimonia, infatti, le buone relazioni tra i Veneziani della città e il principato. Se gli episodi sopra menzionati successero dopo l'arrivo di Caterina in Morea, questo significherebbe che essa aveva certamente delle responsabilità. Se gli avvenimenti si riferiscono invece al periodo di governo di Bertrando des Baux, sembrerebbe in questo caso che l'imperatrice intendeva rimediare alla situazione. Quest'ultima ipotesi è la più plausibile, se si tiene conto del comportamento che il bailo aveva dimostrato nella vicenda di Patrasso. Caterina avrebbe fatto, in questo caso, delle promesse ai Veneziani, che alla fine del 1340 non erano ancora state mantenute. Le prime lettere di protesta sono datate appunto in questo periodo.

L'arrivo di queste lettere a Venezia, riguardanti i danni subiti dai sudditi veneti, fece intervenire il Senato. In particolare, il 7 novembre 1340, furono eletti tre Savi, i quali erano Pancrazio Giustinian, Pietro Miani e Marco Morosini. Questi avevano l'incarico di esaminare le lettere di protesta arrivate da Clarenza e da altre zone della Romania e consigliare per iscritto

${ }^{13} \mathrm{La}$ data si trova in una lettera di Domenico Bonciani a Acciaiuolo Acciaiuoli,padre di Nicolò, scritta il 14 ottobre 1338 a Firenze: J. BuCHON, Nouvelles recherches historiques sur la principauté de Morée et ses hautes baronies, vol. II, Parigi, 1843, pp. 106-108, $\mathrm{n}^{\circ} 13$ : «Sapi che, sabato che passò, a vespero, a di 10 di questo mese, Niccola tuo si partie di qua per andare a buon ora in Romania, molto onorevolmente e molto grandemente...». Un altro documento, datato del 17 luglio 1341 e pubblicato da J. BUCHON, Nouvelles recherches historiques, op. cit., pp. 108-109 è un estratto di un atto, di difficile interpretazione, tra l'imperatrice e il suo consigliere fiorentino. Questo documento si riferisce a un accordo precedente al viaggio dei due in Morea. Nel documento, la data del 15 novembre (settima indizione), che si riferisce al passaggio di Nicolò da Brindisi a Clarenza, è del anno 1338.

«Anuario de Estudios Medievales», 33/2 (2003), pp.905-926 .- ISSN 0066-5061. 
il Senato su come meglio agire ${ }^{14}$. Una deliberazione del 9 gennaio 1341, programmava infine una riunione da farsi, con l'ordine del giorno riguardante i fatti di Clarenza ${ }^{15}$.

Il 13 gennaio, il Senato, esaminando la situazione, su consiglio dei Savi, stabiliva d'inviare un ambasciatore presso Caterina di Valois a Clarenza. L'ambasciatore aveva l'incarico di protestare ufficialmente per i maltrattamenti e i danni subiti dai mercanti veneziani e di chiedere gli indennizzi. In caso di risposta negativa o parziale, egli doveva corrispondere con il Senato, e attendere nuovi ordini ${ }^{16}$. Il Senato decideva inoltre il divieto per le navi veneziane di caricare e scaricare merci nella città di Clarenza, e negli altri porti soggetti all'imperatrice finché la questione non sarà risolta ${ }^{17}$. Il 15 gennaio 1341 l'embargo fu esteso a Patrasso, però per salvaguardare gli affari dei mercanti, furono dispensate le navi vuote, le quali potevano caricare le merci in deposito ${ }^{18}$. L'ammenda per in contravventori dell'embargo fu stabilità al 50 per cento del valore delle mercanzie ${ }^{19}$. Un terzo della somma era da destinarsi al denunziante, se questi aveva detto il vero, un terzo al comune di Venezia, l'altro terzo alla magistratura che aveva l'onere di svolgere l'inchiesta. A Venezia le magistrature incaricate di vigilare sulle merci in partenza dalla città verso le terre dell'imperatrice erano: i Provveditori di comun, gli Ufficiali al cattaver, i Capitani delle poste e gli Ufficiali sopra le mercanzie del Levante. Mentre nella Romania incaricate della vigilanza erano le autorità veneziane sul posto, come per esempio i Castellani di Corone e di Modone.

L'ambasciatore s'imbarcò su una flottiglia, guidata dal capitano Zuane Steno $^{20}$, e aveva con sé un notaio, quattro paggi, un contabile e un cuoco. Egli

${ }^{14} \mathrm{ASV}$, Senato, Misti, reg. 19, f. 41 v. (doc. $\mathrm{n}^{\circ} 1$ ).

${ }^{15}$ ASV, Senato, Misti, reg. 19, f. 51 v. (doc. $\left.\mathrm{n}^{\circ} 2\right)$.

${ }^{16}$ ASV, Senato, Misti, reg. 19, f. 52 r., regesto in Fr. THIRIET, Regestes des délibérations du Sénat de Venise concernant la Romanie, Parigi, 1958, vol. 1, p. 45, $\mathrm{n}^{\circ} 116$.

${ }^{17} \mathrm{ASV}$, Senato, Misti, reg. 19, f. 52 r., 13 gennaio 1341 (doc. $\mathrm{n}^{\circ} 3$ ).

${ }^{18}$ ASV, Senato, Misti, reg. 19, f. 53 r., 15 gennaio 1341 (doc. $\mathrm{n}^{\circ} 4$ ).

${ }^{19} \mathrm{La}$ copia settecentesca del registro 19 del fondo Senato, Misti ci fornisce un importo sbagliato della pena cioè libre cento! Sembra che il copista abbia interpretato in questo modo la lettera L che non sta per libra ma per cinquanta. ASV, Senato, Misti, reg. 19, f. 52 r., 13 gennaio 1341 (doc. $n^{\circ}$ 3): «sub pena .L. pro .C. valoris eorum».

${ }^{20}$ ASV, Senato, Misti, reg. 19, f. 52 r., 13 gennaio 1341 (doc. $\mathrm{n}^{\circ}$ 3). Una ulteriore deliberazione sul movimento delle galee del Golfo e indirizzata al loro capitano, e ci informa che l'ambasciatore si sarebbe imbarcato su di esse con destinazione Clarenza o un altro porto della 
poteva disporre per se stesso e per il suo seguito di 3 soldi grossi al giorno. Il suo salario fu stabilito a 300 lire in quattro mesi ${ }^{21}$.

Il 15 marzo 1341, il Senato aggiornava la commissione all'ambasciatore, incaricandolo di nuovi casi: l'uno riguardante Nicolò Miani, mercante veneziano ${ }^{22}$, e l'altro riguardante il conflitto tra l'arcivescovo di Patrasso e l'imperatrice. L'importanza di quest'ultimo caso pro honore nostro (per Venezia) fu riaffermata nella deliberazione del 17 marzo 1341. Il conflitto dunque persisteva dopo due anni e mezzo di permanenza dell'imperatrice nel principato, nonostante l'intervento papale ${ }^{23}$. L'ambasciatore giunse a Clarenza un po' dopo il 17 marzo e molto prima del 18 giugno, data alla quale il Senato ordinava ai Savi di relazionare sulle lettere che l'ambasciatore aveva già inviato a Venezia ${ }^{24}$. I tre Savi, Marco Morosini, Marco Bragadin e Donato Arimondo ${ }^{25}$, nel vagliare l'operato dell'ambasciata, avevano la possibilità di convocare testimoni sotto giuramento e di imporre sanzioni in caso di irregolarità ${ }^{26}$.

L'ambasciatore, al suo arrivo a Clarenza, aveva con se delle disposizioni scritte (capitula) redatte dal Senato a seguito del parere dei Savi. Egli, dopo aver fatto quanto gli era stato chiesto, soprattutto protestare per i danni subiti dai Veneziani, doveva inviare a Venezia delle lettere con le risposte dell'imperatrice sui vari punti. Il Senato, valutando con i Savi, le risposte dell'imperatrice, prendeva delle decisioni, comunicando poi all'ambasciatore nuove disposizioni ${ }^{27}$.

Morea, ASV, Senato, Misti, reg. 19, f. 68 r., 15 marzo 1341 (doc. $\mathrm{n}^{\circ} 7$ ).

${ }^{21}$ ASV, Senato, Misti, reg. 19, f. 52 v., 15 gennaio 1341 (doc. $n^{\circ} 4$ ). Nella copia il salario del ambasciatore è libras trecentas in quadrigentas in mense!

${ }^{22}$ Questo caso sarà studiato più avanti.

${ }^{23}$ ASV, Senato, Misti, reg. 19, f. 68 v. (doc. $\mathrm{n}^{\circ} 7$ ), il regesto è in Fr. THIRIET, Regestes des délibérations du Sénat, op. cit., vol. 1, p. $45, \mathrm{n}^{\circ} 116$. I contrasti tra le due parti danneggiavano gli interessi commerciali veneziani. I Veneziani avevano buone relazioni con l'arcivescovo Ruggero al punto che nel 27 maggio 1340, durante il conflitto, il Senato dà l'autorizzazione di esportare senza dazi delle armi: «primo lanceas CC bonas et bene ferratas; item paresios CC; item dardos CCCC; item quarellos $X^{m}$ : G. FEDALTO, La chiesa latina, op. cit., vol. 3 , p. 50, n $^{\circ} 98$ Su l'intervento papale, cf. E. Gerland, Neue Quellen, op. cit., p. 27; A. BoN, La Morée franque, op. cit., p. 212.

${ }^{24}$ ASV, Senato, Misti, reg. 19, f. 87 r. (doc. $n^{\circ} 8$ ).

${ }^{25}$ Sono menzionati nella deliberazione del 10 luglio 1341, ASV, Senato, Misti, reg. 19, f. 95 r. (doc. $\left.n^{\circ} 10\right)$.

${ }^{26}$ ASV, Senato, Misti, reg. 19, f. 87 v. (doc. $n^{\circ} 9$ ).

${ }^{27}$ ASV, Senato, Misti, reg. 19, f. 95 r. (doc. $\mathrm{n}^{\circ} 10$ ).

«Anuario de Estudios Medievales*, 33/2 (2003), pp.905-926 .- ISSN 0066-5061. 
Per quanto concerne le iniuria fatte al console veneto di Clarenza, l'imperatrice non diede risposte adeguate, il Senato decise allora di non insistere più sull'argomento. Per i danni subiti dal mercante Marco Gradenigo, l'imperatrice aveva promesso un risarcimento, l'ambasciatore era stato quindi incaricato di chiedere delle garanzie scritte. Altro mercante danneggiato era Schiavo Vitturi. Questi era stato obbligato dalle autorità del principato a scaricare dalla nave e a vendere del frumento al prezzo di 19 sterline per modio al posto del prezzo di mercato di 22 sterline. L'ambasciatore doveva in questo caso chiedere la differenza di 3 sterline per modio per 1500 modii. Francesco e Ricio Loredan potevano testimoniare con giuramento sul danno. Ricio però in quel momento si trovava a Venezia ed era irreperibile, quindi rimaneva solo Francesco che poteva provare giurando sull'accaduto. Anche nel caso di Schiavo Vitturi, se l'ambasciatore non otteneva immediata soddisfazione o garanzie dall'imperatrice, aveva ordine di non insistere oltre. Altro caso riguardava il capitano di Andrusa. Egli aveva provocato danni ai possedimenti veneziani a Corone e Modone. L'imperatrice per questa questione promise di far restituire gli animali e le altre cose portate via. Sulla vicenda dei 100 iperperi del mercante Tomà Bon, abitante di Corfù, l'imperatrice diede risposte soddisfacenti. Sul caso riguardante le valanie di Marco Falier, l'ambasciatore aveva l'incarico di chiedere il risarcimento, o alla peggio di accettare l'arbitrio di sapientes scelti in comune con l'imperatrice. Invece sulla controversia riguardante dei remi sequestrati a Marco Falier, l'ambasciatore doveva chiedere garanzie scritte. Nicolò Miani era stato spogliato delle sue cose, l'ambasciatore in questa questione era incaricato di ottenere la restituzione immediata di queste, o eventualmente di ottenere delle promesse di restituzione. Generalmente il Senato chiedeva, tramite l'ambasciatore, all'imperatrice la reintegrazione dei danni, o alternativamente delle garanzie scritte, o nella peggiore delle ipotesi delle generiche promesse per il futuro.

Nell'estate del 1341, l'imperatrice ritornò in Puglia ${ }^{28}$, lasciando irrisolte alcune delle questioni poste dall'ambasciatore. Per di più se ne aggiunse una di nuova, riguardante il mercante Nicoletto Foscarini, il quale

\footnotetext{
${ }^{28}$ Un documento che si riferisce ai servizi prestati da Nicolò Acciaiuoli in Morea porta la data del 17 luglio 1341 (indizione ottava), stranamente questa data corrisponde alla nona indizione: J. BUCHON, Nouvelles recherches historiques, op cit., pp. 108-109, $n^{\circ}$ XIV. Una conferma si trova nella deliberazione del Senato datata del 23 luglio ASV, Senato, Misti, reg. 19, f. 98 r. (doc. $\left.\mathrm{n}^{\circ} 13\right)$, dove la magistratura si riferisce agli homines imperatricis, come se Caterina non fosse più presente a Clarenza.
} 
aveva subito dei danni di cui noi ignoriamo la natura. Egli affermava di avere delle prove e anche delle testimonianze a sostegno della sua richiesta di risarcimento. Ma i testimoni non si trovavano in quel momento a Venezia. Alcuni di questi erano mercanti di Clarenza ${ }^{29}$.

$\mathrm{Al}$ termine del mandato, l'ambasciatore poteva imbarcarsi sulle galee del Golfo, le quali dovevano tornare dalla missione di scorta alle galee di Romania. Il Senato aveva però dato la possibilità all'ambasciatore di prorogare, se necessario, la sua permanenza a Clarenza e di tornare con la scorta al seguito delle galee di $\mathrm{Cipro}^{30}$. Il ritorno però non equivaleva alla conclusione della sua missione. L'ambasciatore doveva infatti consigliare i Savi sui fatti di Clarenza, i quali erano incaricati di continuare con ampi poteri le indagini soprattutto sul caso Foscarini. Grazie a questa deliberazione è possibile finalmente conoscere il nome dell'ambasciatore, il quale era Tommaso Gradenigo ${ }^{31}$.

L'interdizione commerciale che il Senato aveva imposto sul territorio del principato e su Clarenza, il 19 luglio 1341, era stata sospesa per un anno. Nel frattempo si lavorava per trovare le prove e rintracciare i testimoni per i casi aperti, in particolare quello riguardante Foscarini. Alla conclusione dell'anno, in caso di esiti non soddisfacenti la situazione ritornerebbe come prima $^{32}$. C'è una testimonianza della presenza dei mercanti veneziani nella città di Clarenza qualche mese dopo. Nel 3 novembre 1341, il mercante Conte Venier della parrocchia di Santa Marina aveva potuto incaricare Giovanni Grion della parrocchia di San Eustachio, abitante a Candia (nunc comoranti Candide), di riscuotere da Bruno Baseio e da Michele Betto, 294 iperperi per un credito derivato da una vendita di stoffe ${ }^{33}$.

In due deliberazioni successive del Senato, datate del 17 novembre 1341, si ha notizia che le questioni di Nicoletto Foscarini, di Marco Falier e Nicolò Miani non erano ancora risolte. Il figlio di Marco Falier e alcuni mercanti che potevano testimoniare il vero sul suo caso dovevano recarsi a Venezia con il prossimo passaggio delle galee del Golfo. Il termine cronologi-

\footnotetext{
${ }^{29}$ ASV, Senato, Misti, reg. 19, f. 96 r., 17 luglio 1341 (doc. ${ }^{\circ} 11$ ); f. 97 v., 19 luglio 1341 (doc. $\left.\mathrm{n}^{\circ} 12\right)$ e f. 98 r., 23 luglio 1341 (doc. $\left.\mathrm{n}^{\circ} 14\right)$.

${ }^{30} \mathrm{ASV}$, Senato, Misti, reg. 19, f. 98 r. (doc. $\left.\mathrm{n}^{\circ} 13\right)$.

${ }^{31}$ ASV, Senato, Misti, reg. 20, f. 18 v., 17 novembre 1341 (doc. $\mathrm{n}^{\circ} 15$ ).

${ }^{32}$ ASV, Senato, Misti, reg. 19, f. 97 v., 19 luglio 1341 (doc. $n^{\circ} 12$ ).

${ }^{33} \mathrm{ASV}$, Cancelleria Inferiore, Notai, b. 73, $\mathrm{n}^{\circ} 16$ not. Marino Enzo, protocollo f. 15 r.
} 
co per la presentazione delle prove sui suoi danni, imposto per la fine del mese di novembre fu prolungato di otto giorni dopo l'arrivo delle galee a Venezia, dandogli così il tempo di raccoglierle dagli arrivati ${ }^{34}$. Il 19 dicembre 1342, il Senato elesse tre nuovi Savi: Nicolò Da Mosto, Giacomello Corner e Pietro Morosini, che dovevano ancora esaminare le questioni di Marco Falier e Nicolò Miani ${ }^{35}$. D'ora in poi, la documentazione non ci dà più informazioni su questa ambasciata e per conseguenza non sappiamo se questi mercanti hanno mai avuto gli indennizzi che aspettavano.

Si nota che alcuni dei mercanti coinvolti nelle vicende di Clarenza non sono del tutto sconosciuti. Marco Gradenigo poteva essere parente di Moretto Gradenigo che era tra i firmatari della richiesta di costruzione del fondaco e della chiesa a Clarenza ${ }^{36}$ e forse aveva una parentela anche con l'ambasciatore Tommaso Gradenigo. Tra i richiedenti vi era anche Marco Falier. Suo figlio, cui ignoriamo il nome, era tra i testimoni per i danni subiti da suo padre. Egli era inoltre tra i mercanti di Clarenza che avevano subito danni a causa della perdita della muda dopo la fiera di San Demetrio. I suoi danni ammontavano a ben 3000 iperperi $^{37}$. Francesco Loredan, è menzionato per la prima volta nella richiesta indirizzata al Senato ${ }^{38}$. Da questa data, lo incontriamo più volte fino al 21 luglio 1356, data nella quale il Senato deliberò su una richiesta di diminuzione delle imposte commerciali, formulata dai mercanti e sottoscritta dallo stesso Loredan ${ }^{39}$. Nicoletto Foscarini probabilmente era lo stesso Foscarini che fu nominato capitano e ammiraglio a Clarenza da Roberto di Taranto, divenuto principe di Morea dopo la morte di sua madre Caterina ${ }^{40}$.

I documenti qui presentati danno un pò di luce sul soggiorno dell'imperatrice Caterina di Valois nella Romania e più precisamente a

${ }^{34} \mathrm{ASV}$, Senato, Misti, reg. 20, f. 22 r., 17 novembre 1341 (doc. $\mathrm{n}^{\circ} 16$ ).

${ }^{35} \mathrm{ASV}$, Senato, Misti, reg. 21, f. 3 r., 19 dicembre 1342 (doc. $\mathrm{n}^{\circ} 17$ ).

${ }^{36} \mathrm{ASV}$, Senato, Misti, reg. 18, f. 60 r., 26 agosto 1339.

${ }^{37}$ ASV, Senato, Misti, reg. 17, f. 112 v., 29 novembre 1338, regesto in Fr. THIRIET, Regestes des délibérations du Sénat, op. cit., p. $39 \mathrm{n}^{\circ}$ 81. Cf. A. LAMBropoulou, $O$

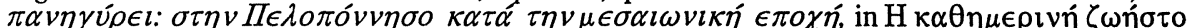

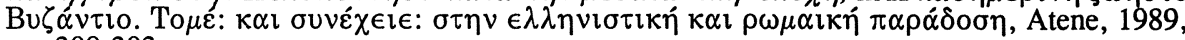
pp. 300-303.

${ }^{38} \mathrm{ASV}$, Senato, Misti, reg. 18, f. 60 r., 26 agosto 1339.

${ }^{39}$ ASV, Senato, Misti, reg. 27, ff. 88 v.-89 r., 21 luglio 1356.

${ }^{40} \mathrm{La}$ nomina è testimoniata in una deliberazione del Senato datata 22 gennaio 1348, ASV, Senato, Misti, reg. 24 , f. 58 r. 
Clarenza, sul procedere dell'ambasciata veneziana, e sulla vita dei mercanti veneziani. Nei documenti non è menzionato il fedele consigliere fiorentino, Nicolò Acciaiuoli. Conoscendo però la sua posizione presso l'imperatrice, possiamo dedurre ch'esso poteva nascondersi dietro le risposte di Caterina. Il ruolo giocato dall'imperatrice, sia nel caso dei Veneziani, sia in quello dell'arcivescovo di Patrasso, era alquanto ambiguo ${ }^{41}$. D'altro canto, Venezia era desiderosa di mantenere a tutti i costi gli straordinari privilegi acquisiti per il suo commercio. Dalle risposte del Senato, si intravede che la finalità di questa ambasciata era sì riuscire ad avere i risarcimenti dei danni, ma più importante era riaffermare i privilegi commerciali e ottenere dall'imperatrice e da suo figlio, nuovi signori della Morea franca, il riconoscimento definitivo di questi.

${ }^{41}$ Notiamo che l'imperatrice aveva fatto arrestare il bailo, Bertrando des Baux a causa del suo violento comportamento per lasciarlo libero poco dopo e ridargli lo stesso posto: A. BON, La Morée franque, op. cit., pp. 212-213.

«Anuario de Estudios Medievales», 33/2 (2003), pp.905-926 .- ISSN 0066-5061. 


\section{DOCUMENTI}

1340 , novembre, 7. Venezia.

Elezione di tre Savi per esaminare le lettere giunte da Creta, Corone e Clarenza

ASV, Senato, Misti, reg. 19, f. 41 v.

Capta.

Quod super litteris Crete, Coroni et Clarentie eligantur tres sapientes per electionem, qui examinatis contentis in eis debeant dare nobis suum consilium in scriptis, cum quo erimus hic et fiet sicut videbitur et expediant se usque ad kallendas ianuarii et quilibet possit ponere partem et vocetur consilium sub pena soldorum decem.

Sapientes electi

ser Pangratius Iustiniano

ser Petrus Miani

ser Marcus Mauroceno

1341, gennaio, 9. Venezia.

Convocazione di una riunione sugli accadimenti di Clarenza

ASV, Senato, Misti, reg. 19, f. 51 v.

Capta.

Quod inducientur hoc factum Clarentie usque ad diem sabbati proximi in mane, qui die et hora vocetur istud consilium, sub pena soldorum decem.

«Anuario de Estudios Medievales», 33/2 (2003), pp.905-926 .- ISSN 0066-5061. 
1341, gennaio, 13. Venezia.

A seguito dell'esame delle lettere, viene deciso l'invio di un ambasceria a Clarenza presso l'imperatrice Caterina di Valois. Imposto un embargo sui domini imperiali.

ASV, Senato, Misti, Reg. 19, f. 52 r.

Regesto: Fr. THIRIET, Régestes des delibérations du Sénat I, p. 45 n 116

Capta.

Examinatis et cognitis iniuriis, offensionibus et damnis illatis nostro consuli Clarentie et nostris mercatoribus, tam in Clarentia, quam in aliis partibus principatus Achaye per officiales et ministros domine imperatricis Constantinopolis ac visis et intellectis litteris eiusdem domine imperatricis et nostrorum castellanorum Coroni et Mothoni, habita collatione cum mercatoribus utentibus in dicto principatu ac cum consule antedicto et super omnibus habita deliberatione. Consulunt concorditer sapientes ut predicta omnia dicte domine imperatrici seriosius et distinctius exponantur et ut in premissis iustius et excusabilius moveamur et ut melius et facilius de premissi satisfactionem et emendam debitam consequamur quod mittatur unus ambaxator ad dictam dominam imperatricem, qui vadat cum presenti armata de qua est capitaneus ser Ioannes Steno et exponat eidem domine predictas iniurias, offensiones et damna per gentem suam nostro consuli et aliis nostratibus irrogata. Et qualiter miramur et turbamur merito dicere, quod nostri fideles in partibus suis sic damnificentur et iniuriose tracentur et de damnis et iniuriis eorum satisfactio et iustitia denegetur. Et per ipsum ambaxatorem emenda predictorum et congrua satisfactio postuletur et si obtinere poterit satisfactionem integram bene quidem, sin autem non posset in totum sed in parte, vel aliter compositionem aliquam nobis utilem inveniret, tunc scribat huc quidquid habebit et suum consilium et mandatum nostrum ibidem prestoletur.

Capta.

Non sinceri .i./ non .O./ alii de parte.

Quod ex nunc sit strictum et cridatum, quod aliquod navigium, quod recedat de hinc, non possit portare ad partes Clarentie et principatus subpositas domine imperatrici aliquas mercationes et res sub pena .L. pro .C. valoris eorum, que portarent. De parte 35.

Nota quod die XIII ian. proclama fuit in Rivoalto ista pars.

Quod ambaxator vadat, ut captum est, et si optinere non poterit commissa, rescribat que fecerit et suum consilium et post modum habita informatione provideatur 
et fiat sicut melius videbitur, habendo ambaxatore in credentia et sic habeatur hic de eo quod ambaxator debet rescribere. Non sin. .1./ De parte .i9.

Pars Ser Petri Civrano Consiliarii.

1341, gennaio, 15. Venezia.

Deliberazione sui salari dell'ambasceria. Si conferma l'embargo e si deliberano le pene e le competenze delle varie magistrature.

ASV, Senato, Misti, reg. 19, ff. 52 v.-53 r.

Capta.

Quod iste ambaxator iturus ad dominam imperatricem habeat secum unum notarium, quatuor famulos, unum expensatorem et unum cochum in dicta ambaxata et possit expendere pro expensis de ore tantum pro se et dicta sua familia soldos tres grossorum pro quolibet die. Et habeat dictus ambaxator pro tota dicta via et ambassiata pro suo salario libras .CCC. in quatuor mensi et ab inde in antea habeat in ratione soldorum viginti grossorum in mense. //

Capta.

Quod ex nunc et statim strictum sit quod nullus Venetus vel fidelis Venetiarum in cetero possit vel debeat portare vel mittere per se vel alium aliquo modo vel ingenio aliqua mercimonia vel alias res de hinc, nec de aliqua alia parte per mare Clarenciam vel aliquas partes principatus subiectas domine imperatrici, nec etiam Patras, sub pena librarum .L. pro .C. valoris eorum, que aliqualiter portarentur vel mitterentur, exceptis navigiis nostrorum vacuis que ire possint ad dictas partes ad levandum mercimonia nostrorum, que ibi essent, que exigende et inquirende committantur extra Venetias omnibus nostris rectoribus et capitaneis et in Venetiis Provisoribus communis, Cattaveris, Capitaneis postarum, et Officialibus Levantis. Et qui accusaverit, si per eius accusationem veritas habebitur habeat tertium condemnationis, tertium commune, tertium rector, capitaneus vel officialis, cui primo facta fuerit accusatio.

Non sin. .3./ non .5./ alii de parte.

Nota quod die XV ian. proclamata fuit in Rivoalto. 
1341, marzo, 15. Venezia.

Notizie sull'invio dell'ambasceria.

ASV. Senato, Misti, reg. 19, f. 68 r.

Cum vir nobilis Marcus Paulo missus fuerit cum galeis Culphi in Siciliam pro facto frumenti cum pecunia nostri communis et ipse non potuerit adimplere intentionem nostram et providendum sit de reditu suo cum pecunia antedicta, Vadit pars, quod committatur capitaneo Culphi, quod in ista prima via sua vadat ad levantum dictum Marcum cum pecunia communis, qui debet expectare Messane. Et si dictus Marcus impeditus forte aliqua de causa venire non posset, recipiat capitaneus pecuniam communis, quo facto redeat versus partes Romanie, deponendo nostrum ambaxatorem, qui vadit ad dominam principissam vel Clarentie vel alibi in ipsis partibus, unde comodius ire poterit ad principissam predictam et inde vadat Coronum vel Mothonum et dimittat dictam pecuniam in manibus castellanorum et deponat ibi nobilem virum Andream Cornario iturum ducham Crete et levet virum nobilem Nicolaum de Priolis, nunc ducham ibi, si venire voluerit et alios rectores qui complevissent et postmodum inde redeundo, faciat viam versus Nigropontem et det et consignet nostro baiulo et consiliariis Nigropontis pecuniam, quam sibi mittimus, quam dicto capitaneo fecimus dari, silicet libras trecentas grossorum, et postmodum redeat Coronum ad accipiendum dictam pecuniam et inde redeat versus partes Culphi, faciendo deponi ducham et rectores et ser Marcum Paulo, si erit cum galeis Ragusii, quando videbitur ipsi capitaneo, quod id comode facere possit et, si dictus ser Marcus non esset cum galeis, dictam pecuniam communis consignet in manibus ser Nicolai de Priolis predicti et postmodum vadat ad suam custodiam. Verum licet predicta omnia sic dicta sint, tamen ea et de via Sicilie et aliis predictis omnibus, sic volumus et ordinamus, quod non obstantibus que dicta sunt, si capitaneus haberet talia nova, per que videretur ei aliud vel aliter providere et facere, relinquimus in sua libertate in onibus et per omnia, eundi et faciendi, quando et sicut ei videbitur pro honore nostro secundu, ea que de novo haberet, quia plura posset idem capitaneus habere et scire de extra que hic non possumus scire.

Non .i./ non sic. .0./ alii de parte. 
1341, marzo, 15. Venezia.

Commissioni per l'ambasciatore sui danni dei Veneziani nel Principato. Notizie sui casi di Nicolò Miani e sul conflitto tra l'imperatrice e l'arcivescovo di Patrasso.

ASV, Senato, Misti, reg. 19, f. 68 v.

Die $\mathrm{XV}^{\mathrm{a}}$ marcii.

Capta.

Quod expediatur ambaxator noster iturus Clarentiam ad dominam principissam, cum hiis que habentur usque modo et cum illis claritatibus que haberi poterunt in factis, quousque galee erunt hic et id quod inde habebit, rescribat nobis et suum consilium et expectet nostrum mandatum. Et si inveniret quod aliqui nostri fideles essent ibi indebite gravati vel damnificati, faciat in favorem eorum id boni quod poterit pro nostro honore. Et committatur etiam sibi factum ser Nicolai Miani de argento sibi accepto in terris dicte domine principisse. In super si concordia facta non esset inter dictam dominam principissam et archiepiscopum Patracensem et ipse videret, quod posset operari aliquod bonum pro concordia partium ipsarum ad id det operam cum honore nostro, sicut ei videbitur.

Non .4./ non sinc. .4./ alii de parte.

1341, marzo, 17. Venezia.

Che l'intervento sulla questione tra l'arcivescovo e l'imperatrice non comprometta l'originale scopo della missione diplomatica .

ASV, Senato, Misti, Reg. 19, f. 68 v.

Regesto: Fr. ThIRIET, Régestes des délibérations, I, p. $46 \mathrm{n}^{\circ} 125$

Die $\mathrm{XVII}^{\circ}$ marcii.

Capta.

Cum pridie foret captum in isto consilio, quod committeretur nostro ambaxatori ituro Clarentiam, quod si postquam erit in partibus illis, videbitur sibi, quod posset aliquid boni procurare inter dominam imperatricem et archiepiscopum Patracensem pro concordio inter eos, quod in hoc faceret, sicut sibi videretur pro honore nostro. Et hoc posset nimis large intelligi et alia nostra principalia negotia

«Anuario de Estudios Medievales», 33/2 (2003), pp.905-926 . - ISSN 0066-5061. 
possent de facili sinistrari, Vadit pars, quod interpositio dicti nostri ambaxatoris de dicto concordio, ut pridie fuit hic captum, intelligatur solum de mittendo litteras, non recedendo de loco, ubi erit idem ambaxator noster, pro factis aliis sibi commissis.

1341, giugno, 18. Venezia.

Commissioni ai Savi, eletti per i fatti di Clarenza.

ASV, Senato, Misti, reg. 19, f. 87 r.

Capta.

Quod Sapientes electi pro factis Clarentie videant et examinent litteras missas nobis per nostrum ambaxatorem et super eis dent nobis suum consilium in scriptis et habeant libertatem ponendi partem, sicut habebant sapientes primo electi.

1341, giugno, 23. Venezia.

Ulteriore commissione ai Savi per i fatti di Clarenza.

ASV, Senato, Misti, reg. 19, f. 87 v.

Quod Sapientes deputati pro factis Clarentie habeant libertatem, quia necesarium est ponendi personas ad sacramentum et imponendi penas sicut eis videbitur pro bono dictorum negotiorum.

1341, luglio, 10. Venezia.

Deliberazione sulle vicende di Clarenza, a proposito dei danni subiti dal console, dal mercanti Marco Gradenigo, Schiavo Vitturi, Tommaso Bon, Marco Falier e Nicolò Miani, e sui danni subiti nei territori di Corone e Modone a causa del capitano di Andrusa.

«Anuario de Estudios Medievales», 33/2 (2003), pp.905-926 .- ISSN 0066-5061. 
ASV, Senato, Misti, reg. 19, f. 95 r.

Regesto: Fr. THIRIET, Régestes des deliberations du Sénat I, p. $47 \mathrm{n}^{\circ} 131$.

Sapientes: ser Marcus Mauroceno

ser Marcus Bragadin

ser Donatus Arimondo

Infrascripte sunt partes super finali responsione domine imperatricis Constantinopolis ad ambaxatores nostri ambaxatoris Clarentie facte super capitulis infrascriptis, continentes formam, in qua contentamur.

Capta.

Et primo super primo capitulo de iniuria facta nostro consuli et cetera. Quod licet tante iniurie nostri communis non sit debite satisfactum scribatur ambaxatori, quod contentur et stetur emende et satisfactioni per dominam imperatricem exibite, demonstrando tamen quod huiusmodi satisfactio satis est levis respectu iniurie irrogate, procurando instanter ne talia imposterum perpetrentur.

De non .i./ non sinc. .13./ alii de parte.

Capta.

Super secundo capitulo ser Marci Gradonico de damno suo, et cetera. Cum domina imperatrix obtulerit in responsione sua facere fieri satisfactionem et emendam dicto ser Marco de suo damno, quod stetur et contentetur de eo quod dicta domina obtulit, habendo ambaxatore illas scripturas, cautellas et securitates, quas ambaxator habere poterit et viderit opportunas, ut servetur et adimpleatur, quod offertur per dominam antedictam.

Capta.

De non .i./ non sinc. .2./ alii de parte.

Super tertio capitulo ser Sclavi Vituri de suo damno et cetera. Quod scribatur ambaxatori, quod obtinere procuret instanter satisfactionem et emendam in tantum quantum est, sterlini tres pro quolibet modio de illis mille quingentis modiis, que in compulsus discaricavit in terram et vendidit ibi, habendo solum sterlinos .XVIIII. pro quolibet modio, nam volebat sterlinos .XXII. pro quolibet modio, quod iustum erat. Et si hoc videtur non posse obtinere, tunc dicat domine imperatrici vel ei qui loco eius foret, quod quia dicta domina asservit ser Franciscum Lauredano et ser Ricium Lauredano scire factum frumenti dicti ser Sclavi, dictus ser Franciscus dato sibi sacramento de veritate dicenda, interogatus et examinatus est super dicto facto per cuius testificationem apparet clare et liquide, quod dictus ser Sclavus per vim et violentiam descaricavit in terram et vendidit frumentum predictum, habendo de modio solum sterlinos XVIIII, cui testificationi deberet dicta domina credere et de ipsa merito contentari, postquam dixit dicta domina dictum ser Franciscum scire veritatem predictorum. Et quod dictus ser Ricius eo quia absens est a Venetiis, examinari non potuit, et quod testificatio dicti ser Francisci mittatur ambaxatori, quam si domina vel alius qui loco eius esset, videre vellet sibi exhibeat et demonstreret. Et si finaliter in 
facto dicti ser Sclavi ambaxator, quod dictum est obtinere non posset, non stet propterea quin compleat alia que dicta sunt et dicentur et Venetias revertatur.

De non .3./ non sinc. .3./ alii de parte.

Super quarto capitulo de damno damnificatorum Coroni et Mothoni per capitaneum Drusum et cetera. Quod postquam restituta sunt animalia ablata et alia quecumque reperientur ablata, restitui facere promittat domina antedicta contentetur et stetur, de satisfactione huiusmodi, procurando ambaxatore illas promissiones et securitates, que ei pro premissorum observantia videbuntur.

Super quinto capitulo Thome Bono mercatoris in Corfù, qui soluit yperperos .C. pro nominibus sibi captis et cetera contentandum est de responsione domine imperatricis, cum satis rationabilis videatur et maxime postquam offert dicta domina facere restitui dicto Thome quidquid invenietur dicto Thome indebite fore ablatum.

Non .i./ non sinc. .4./ alii de parte.

Super sexto capitulo ser Marci Faletro de facto sue valanie et cetera. Quod scribatur ambaxatori, quod procuret obtinere emendam et satisfactionem dicte valanie et si finaliter obtinere non posset contentetur de stando iuri et iustitie, secundum arbitrium sapientum communiter eligendorum sicut offert dicta domina. Super facto vero remorum ablatorum eidem ser Marco stetur et contentetur de oblatione facta per dictam dominam, procurando ambaxatore habere scripturas et promissiones quas poterit et quas viderit expedire, ut promissa serventur.

Non .2./ non sinc. .3./ alii de parte.

Super septimo capitulo ser Nicolai Miani de suis rebus et cetera. Quod mandetur ambaxatori, quod procuret instanter habere et recuperare dictas res et si finaliter obtinere non posset, contentetur de responsione dicte domine, que dicit, quod pro honore domini ducis et eius Communis quidquid ad ea perveniet de bonis predictis, restitui faciet predicta ser Nicolao, cum ad eam vel eius curiam nichil pervenerit de bonis predictis, quia officiales, qui eas res accepit nundum fecit suum computum procurando ambaxatore habere illas cautelas et securitates, quas poteri, et crediderit pro predictorum observantia expedire.

De non .2./ non sinc. .4./ alii de parte.

1341, luglio, 17. Venezia.

Commissioni ai Savi sul caso Foscarini.

ASV, Senato, Misti, reg. 19, f. 96 r.

Die eodem.

Capta.

«Anuario de Estudios Medievales», 33/2 (2003), pp.905-926 .- ISSN 0066-5061. 
Quod committatur Sapientibus pro ambaxata Clarencie, quod possint recipere testes cum iuramento in facto de chà Fuscareno, tam illos quos voluerint illi de chà Fuscareno, quam mercatores Clarencie.

1341, luglio, 19. Venezia.

Deliberazioni sulla vicenda di Nicoletto Foscarini, e sull'embargo.

ASV, Senato, Misti, reg. 19, f. 97 v.

Consil.: ser Andreas Bondemiro ser Andreas Mauroceno ser Andreas Maripetro ser Nicolaus Venerio

Capit.: ser Marcus Longo ser Nicolaus Lion ser Leonardus Contareno

Capta.

Cum ser Nicoletus Fuscareno dicat se habere probationes et testes, qui non sunt hic, per quos probabit clare damnum suum, Vadit pars, quod ipse probationes et testes, quando venerint recipiantur et nichilominus scribatur nostro ambaxatori, quod obtineat et compleat super aliis capitulis sibi commissis, sicut captum est, et de damno etiam dicti ser Nicoleti quousque ibi erit procuret quidquid boni poterit. Et expedictus ambaxator Venetias revertatur, verum aperiatur, quod mercatores cum suis bonis possint ire Clarenciam, non obstante processu et receptis probationibus dicti ser Nicoleti redeunde ambaxiatore venietur huc cum eo quod habebitur super facto dicti ser Nicoleti et providebitur pro suo favore et conservatione sui iuris sicut videbitur huic consilio et si de eo quod fuerit cognitum per istud Consilium quod iuste habere debeat de suo damno non fuerit satisfactum eidem vel contentus usque ad unum annum proximum. Ex nunc captum et firmatum sit quod elapso anno predicto, nostri non vadant, nec utantur, nec mittant in Clarencia, nec in terris et locis suppositis domine imperatrici, sub penis et conditionibus contentis in processibus Clarencie.

Non sinc. .9./ de non .21./ de parte .33. 
1341, luglio, 23. Venezia.

Deliberazione sul ritorno dell'ambasciatore.

ASV, Senato, Misti, reg. 19, f. 98 r.

Die XXIII iulii.

Capta.

Quod scribatur ambaxatori Clarentie, quod obtinendo et non obtinendo, que sibi scribimus Venetias revertatur et scribatur capitaneo Culphi, de levando ipsum ambaxatorem et conduci faciat Ragusium postquam fecerit scortam galeis Romanie et redierit ad partes Culphi. Et insuper scribatur dicto ambaxatori, quod nullo modo recipiat in solutionem alicuius nostri fidelis de bonis et rebus Nicoleti Fuscareno sibi acceptis per homines imperatricis. Verum si videretur ambaxatori, quod mora sua esset fructuosa pro factis, habeat libertatem indutiandi reditum suum, ad rediendum cum dictis galeis Culphi postquam fecerunt scortam galeis Cypri.

1341, luglio, 23. Venezia.

Comissioni ai Savi sul caso Foscarini.

ASV, Senato, Misti, reg. 19, f. 98 r.

Capta.

Quod sapientes deputati pro factis Clarentie debeant et possint accipere testes et testificationes et eos et eas examinare quos et quas producere voluerit ser Nicloletus Fuscareno in favorem iurium suorum.

1341, novembre, 17. Venezia.

Commissioni ai Savi e all'ambasciatore di esaminare i fatti di Clarenza. L'ambasciatore è Tommaso Gradenigo.

ASV, Senato, Misti, reg. 20, f. $18 \mathrm{v}$

«Anuario de Estudios Medievales», 33/2 (2003), pp.905-926 .- ISSN 0066-5061. 
Capta.

Quod committatur Sapientibus alias electis pro factis Clarentie propter reditum nostri ambaxatoris de ipsis partibus, quod examinent conditionem negotiorum et dent nobis suum consilium in scriptis, cum quo erimus hic et fiet sicut videbitur et possint de novo recipere testes et probationes quos et quas recipere voluerint illi de chà Fuscareno producere et alii et propterea possint ponere penam et penas et personas ad sacramentum et quilibet possit ponere partem.

Ser Thomas Gradonico ambaxator.

Capta.

Quod ambaxator et rectores qui nuper redierunt cum galeis debeant esse cum Sapientibus, qui debent eligi, scilicet quilibet cum illis qui habent consulere de factis, de quibus sunt informati et possint in eis ponere partem, sicut possunt Sapientes.

1341, novembre 17. Venezia.

Prorogato il termine per la presentazione delle prove sul caso di Marco Falier.

ASV, Senato, Misti, reg. 20, f. 22 r.

Capta.

Cum filii ser Marci Faletro et alii plures mercatores qui sciunt veritatem de facto damni dati ser Marco predicto in Clarentia, non venerint Venetias ad huc sed expectentur cum galeis Culphi, que in proximo sunt venture. Et propterea idem ser Marcus non possit probare dictum damnum infra terminum sibi datum per istud consilium, qui terminus est per totum mensem presentem novembris, Vadit Pars, quod considerata predicta, dictus terminus prorogetur sibi per dies octo, post adventum Venetias galearum ipsarum. Et si consilium et cetera.

1342, dicembre, 19. Venezia.

Elezione di tre Savi per i casi di Marco Falier e Nicolò Miani.

ASV, Senato, Misti, reg. 21, f. 3 r.

Capta.

«Anuario de Estudios Medievales», 33/2 (2003), pp.905-926 .- ISSN 0066-5061. 
Quod pro facto ser Marci Faletro et ser Nicolai Miani qui iam diu conquesti sunt de suis damnis sibi illatis subditos domine imperatricis Clarentie, que fuerunt requisita per nostrum ambaxatorem et sunt super inde responsiones et partes capte et alie scripture. Eligantur per electionem tres Sapientes qui examinent predicta et omnia ad factum pertinentia et dent nobis suum consilium in scriptis, cum quo erimus hic et fiet sicut videbitur et quilibet possit ponere partem et habeant terminum per totum mensem ianuarii proximi.

Sapientes: ser Nicolaus de Musto, ser Iacobellus Cornario, ser Petrus Mauroceno sancti Moysi. 